\title{
Study on Electronic Records Filing of Mobile Terminal
}

\author{
Yu-fen JIANG, Ping WANG \\ Jiangsu Archives
}

\begin{abstract}
Many of electronic records produced by mobile terminal are important information resources for individuals and society, which have preservation value and are a treasury of archives. However, mobile terminal updates quickly, tends to lose data and has security risks; it is not suitable for long-term preservation of electronic records. The article analyzes the characteristics of electronic records of mobile terminal, current management status and problems, and discusses the key issues of electronic records of mobile terminal filing.
\end{abstract}

Keywords-Mobile terminal; Electronic records of mobile terminal; Filing; Metadata

\section{INTRODUCTION}

The earliest and most comprehensive records in the real life of today's world are mostly recorded by mobile terminals. Many of them are the original records directly formed by people in various social activities. They are not only the content of personal work and life, but also the witness of social development. The mobile terminal stores a large number of electronic files in various forms such as text, pictures, audio, video, etc., which has preservation value for individuals or society, and is a treasure trove of files. However, the mobile terminal itself is not suitable for long-term preservation of electronic documents; they are faced with the risk of fleeting, or may be improperly disclosed or utilized in inappropriate situations. Therefore, study of the filing of electronic records of mobile terminal is particularly important.

\section{MOBILE TERMINAL AND MOBILE TERMINAL USER}

\section{A. Mobile terminal}

A mobile terminal or mobile communication terminal refers to a computer device that can be used in mobile. The broad definition includes a mobile phone, a notebook, a tablet computer, a POS machine, and even an on-board computer. In this paper, a narrow definition is used to refer to smart phones and tablets with multiple application functions ${ }^{[1]}$.

Modern mobile terminals already have strong processing power, large capacity memory and solidified storage media, and a stable operating system. They are a complete ultra-small computer system that can perform complex processing tasks. At the same time, mobile terminals have a very rich communication method, which can communicate through mobile 4G, wireless LAN, Bluetooth and infrared. From hardware, software, communication capabilities and functional use, mobile terminals have evolved from simple call tools to an integrated information processing platform. The performance of mobile terminals is getting higher and higher, including multimedia data collection, large-capacity storage, etc., and its

Fund: Part of the research results of the National Archives Science and Technology Project "Electronic records of mobile terminal collection, filing and long-term preservation research”. functions are more and more abundant to be applied to various aspects such as office, study and life.

\section{B. Mobile terminal users}

According to the 41st Statistical Report on the Development of China's Internet Network issued by China Internet Network Information Center (CNNIC) ${ }^{[2]}$, as of December 2017, the number of Internet users in China reached 772 million, and the penetration rate reached $55.8 \%$. The proportion of Internet users using mobile phones has increased from $95.1 \%$ in 2016 to $97.5 \%$. The usage rate of desktop computers, laptops and tablets has declined. Mobile phones continue to crowd out the use of other personal Internet devices. The diversity of mobile terminals makes it easy for users to process information anytime and anywhere using fragmentation time, which is one of the most widely used electronic devices with a large number of electronic files.

\section{ELECTRONIC FILES FORMED BY MOBILE TERMINALS}

The mobile terminal has the advantage of sharing, opening and interacting with the Internet anytime and anywhere. Compared with the traditional media, it integrates various elements such as text, pictures, audio, video, etc., and integrates various forms of current electronic files. According to the definition in the Filing and Management Regulations for Electronic Documents (GB/T 18894-2002), electronic files can be divided into five categories: text files, graphic files, image files, audio files and video files. In addition to this, there are other types of electronic files, such as various types of analysis, calculations, tests, design parameters, and management data, including both exported data in a relational database, formatted package data, data files such as 3D data and spatial geographic data generated by professional software, as well as computer program files such as commercial or self-developed system software, application software, and related supporting software.

The book Mobile Internet: Terminals, Networks and Services mentioned that mobile terminal research mainly includes two aspects of technical research, one is terminal hardware, operating system, software platform and application software; the second is energy saving, positioning, context awareness, Content adaptation and human-computer interaction ${ }^{[3]}$. The fundamental purpose of electronic document classification is to maintain the relationship between the document and the business activities that produce the document. Therefore, the classification of electronic files generated by mobile terminals based on powerful application functions and unique social attributes also has certain particularities. It can also be categorized as follows: 
application installation package, instant messaging for social software, life record class, mobile office class, map and location information class and so on.

\section{Management StAtus OF ELECTRONIC RECORDS OF MOBILE TERMINAL}

With the development of mobile internet technology, mobile terminal devices are widely used in government official activities and public daily life. At present, the management of the life cycle of collection, filing, storage and utilization of electronic records of mobile terminals has not been fully studied and practiced in related fields.

\section{A. From an institutional perspective}

China's archives management department is basically in its infancy in the management of electronic records of mobile terminal. Domestic file mobile terminal services are mainly manifested in the development and promotion of archive resources ${ }^{[4]}$, including public service functions, one is the introduction of archives institutions, and the other is business processing, that is, file search business and reservation business. The file publicity function is mainly realized through the file management APP to publish electronic exhibitions and electronic editing and research, through which users can search and access relevant information; interactive function mainly includes functions such as message and interaction, and realizes the initiative and personalization of information push through the interactive platform. The above functions belong to the mobile terminal file service implemented by the more popular file management departments using mobile Internet applications such as APP and WeChat public number. However, in the field of management of massive electronic files formed by mobile terminals, there are issues like "whether or not to incorporate such information into the document framework, how to distinguish public and private boundaries, how to determine the preservation value of electronic messages, how to effectively manage such information, how to ensure information security and personal privacy", although it has received attention, no basic management system and filing mechanism have been established yet.

\section{B. From users'perspective}

As a device for storing and managing electronic files, mobile terminals have a large security risk. Although the mobile terminal is convenient in using, it is easy to be lost and all electronic files will be lost at the same time. Due to the fast update of its application system, major updates often result in the loss of related electronic files that were originally saved. Compared with computers, mobile phone systems are less secure and vulnerable to malicious programs such as viruses. There are also significant limitations to the method of collecting, filing and long-term preservation of electronic documents by transferring valuable electronic files stored in the mobile terminal to the computer. Mobile terminal users are far greater than computer users, and many people do not have the habit and awareness of using computers to manage electronic files in mobile terminals. Mobile terminals and computers use different operating systems. Some electronic files in mobile terminals may not be able to open and read in the computer, which makes filing and long-term storage difficult. In addition, since the mobile terminal device is limited by hardware conditions, it can store more limited capacity. As the amount of stored data gradually accumulates and reaches the storage limit at a certain time, this situation not only affects the performance of its implementation, but also causes the loss and damage of precious data. Users may store some electronic files in the public cloud, which reduces the running burden of the device. However, based on the public cloud of the third party, users have weak control over their data, and data is vulnerable to natural disasters, human factors or legal norms, resulting in data loss ${ }^{[5]}$.

\section{KEY ISSUES OF ELECTRONIC RECORDS OF MOBILE TERMINAL} FILING

\section{A. Filing format}

Electronic files are the basis of the information resources of mobile terminals, and they are of various types and formats. Electronic Document Filing and Electronic File Management Specification GB/T18894-2016 stipulates that "electronic documents shall be formed, collected and filing in a common format, or converted to a common format before filing." At the same time, the electronic file filing format should have the performance like open format, no binding on hardware and software, convertible and others.

In the massive electronic files of mobile terminal devices, image-like electronic files have a large proportion. On the one hand, due to the better and better shooting function of modern mobile terminals, the quality of image presentation is getting higher and higher; on the other hand, due to the portability of mobile terminals, smart phones play an important role in daily photography. As a multimedia electronic file filing, the image file must guarantee its originality. In theory, image files should be saved in an uncompressed or lossless compressed image format. However, when an image file is saved in an uncompressed or lossless compressed image format, a large number of image files will take up a large amount of storage space, leading to less operability. In fact, it is the perfection and de velopment of compression technology that makes digital storage and network transmission possible. Therefore, the image file filing should take into account the originality and the space occupied by the image file disk.

In addition, the variety of operating systems and application software of mobile terminals and frequent upgrades have brought challenges to image file readability, which requires an open format with a higher degree of standardization when the image file is filing, mainstream format that more software tools and more widely used in the industry and portable format that is highly independent of the technical environment and has good compatibility with different application systems.

\section{B. Metadata standard}

Metadata is data that describes the background, content, structure, and overall management of a file. Metadata can fully describe electronic files, ensure their authenticity, completeness and availability, support the organization and query of file information, support the integration and optimization of management processes, and facilitate the full control of electronic files. Electronic document management metadata is the "lifeline" of electronic document management, 
and it is the indispensable key to guarantee the value of electronic documents in electronic document management, which has become the consensus of the international document and archives field.

Information and Documentation File Management Process File Metadata Part 1: Principle (GB/T 26163) regulates the creation, management and application of metadata. In 2009, 2014 and 2017, the National Archives Bureau successively issued the Metadata Program for Electronic Documents of Documents (DA/T46-2009), the Metadata Program for Photo-type Electronic Files (DA/T54-2014) and Audio Recording Video File Metadata Program (DA/T 63-2017). The provinces and cities have also introduced metadata management programs. The number of metadata elements in the program is relatively large. According to the survey, taking the document file metadata program as an example, there are 132 in Jiangxi Province, 84 in Liaoning Province, 63 in Tianjin, and 84 in Qingdao.

Not only the Filing format of each type of electronic file formed by the mobile terminal device is specified, but also a metadata scheme is separately formed to ensure the integrity, usability, authenticity and security of the electronic file. At present, China has not yet developed a metadata standard scheme for electronic records of mobile terminals. The standard should fully consider the characteristics of image-based electronic files formed by mobile terminals, combined with the Photographic Electronic Archives Metadata Program (DA/T54-2014) issued by the National Archives.

\section{Authenticity guarantee}

The electronic files of the mobile terminal are basically stored locally and stored in the terminal device. During the collection, filing and long-term preservation of the electronic records of mobile terminal, the information security risks that the electronic file may face mainly come from the following aspects:

\section{1) Cybersecurity risk}

Network security is an important aspect of information security. On the one hand, the network structure and network facilities are extremely complex, and electronic files face many security risks in the process of storage, transmission, management and network-based service provision of mobile terminals. The mobile terminal is faced with a complex network environment and is exposed to threats such as viruses, hackers, illegal intrusions, unauthorized access, etc., which will greatly affect the security of electronic file circulation ${ }^{[6]}$.

\section{2) Data security risk}

In the process of collecting filings, electronic documents are subject to data security risks caused by tampering with the contents of electronic documents, disclosure of electronic file information, and destruction of electronic file information. The data security risks faced by electronic files can lead to the destruction of the security attributes such as authenticity, integrity, readability and usability of electronic file data.

\section{3) System security risk}

The security of the mobile terminal information system is the cornerstone for the normal use of electronic files. It is composed of the hardware and software of the mobile terminal and supports the generation, transmission, storage and utilization of electronic files. Besides, it is a digital environment necessary for electronic files. Viruses, logic bombs, hardware and software damage, force majeure, man-made malicious attacks and other factors may cause the system to be destroyed, resulting in the destruction of the security attributes of electronic files built on the system and unusual working of electronic files.

In the face of more and more information security risks, it is necessary to technically improve the security management performance of electronic records of mobile terminals, and take active defense. Advanced data encryption, digital signatures, digital certificates, digital abstracts, digital watermarking, information hiding, and CA authentication techniques $^{[7]}$ should be used to ensure the authenticity and reliability of the contents of electronic documents and the security and confidentiality of electronic documents during collection, storage and transmission and prevent illegal access and arbitrary changes to electronic files. With the maturity, popularity and new technology of these technologies, the originality and authenticity of electronic documents can be more reliable and effective.

\section{CONCLUSION}

The effective filing of electronic records of mobile terminal helps to record social activities, build personal and collective memories, ensure the authenticity and security of electronic documents, promote the sharing of resources and improve the efficiency of personal electronic files and electronic files. Strengthening the filing of electronic records of mobile terminal, and realizing the long-term preservation and sharing of filing electronic files using mobile Internet are important means of archival information construction, which will help spread the archives knowledge to the whole society, enhance the public's awareness of file participation, and promote the socialization of archives work.

\section{REFERENCES}

[1] Baidu Encyclopedia, mobile terminal, https://baike.baidu.com/item/\%E7\%-A7\%BB\%E5\%8A\%A8\%E7\%BB \%88\%E7\%AB\%AF\#1, July 2, 2018.

[2] Statistical Report on the Development of China's Internet Network, China Internet Network Information Center (CNNIC). January 2018.

[3] Luo Junzhou, Wu Wenjia \& Yang Ming. Mobile Internet: Terminal, Network and Service[J]. Chinese Journal of Computers. 2011(11): 2022.

[4] Zhou Yaolin \& Jia Congcong. Research on the Development Strategy of Digital Case Information Service under the "Internet +" Strategy[J].Archives and Communications, 2016 (04) :(56-61).

[5] Hu Changping \& Huang Shushu. User Rights Protection in Public Cloud Storage Service [J]. Information Theory and Practice. 2016(11): 17-21.

[6] Dong Jingjing, Huo Shanshan, et al. Research on Information Security Technology of Mobile Office Terminals [J]. Computer Technology and Development. 2018 (01): (155-158).

[7] Jiang Yufen. Research on Filing of Electronic Documents in Trusted Environment[J]. Archives and Construction. 2016(04):(33-34). 